\title{
Use of electromyography to study leg muscle activity in patients with arthritis and in normal subjects during rising from a chair
}

\author{
J. S. MUNTON, M. I. ELLIS, AND V. WRIGHT \\ From the Rheumatism Research Unit, University Department of Medicine, General Infirmary at Leeds
}

SUMMARY A previous study indicated the need for patients with arthritis to have an armchair from which it is easy to rise. To determine criteria for such a chair a greater understanding of the rising activity and the objective assessment of chair design is required. The major muscle groups of the leg were monitored by electromyography (EMG) in normal subjects and for patients with arthritis during rising from a chair. The effects on EMG patterns of changes of seat height, foot position, and the use of armrests were studied. This paper outlines the practical difficulties that must be borne in mind when designing an EMG study on arthritic and elderly subjects. The results did not illustrate any differences in the pattern of muscle activity between arthritic.and normal subjects, nor did they show any differences caused by changing the variables of chair design.

It became necessary to study the activity of leg muscles while rising from a chair during an investigation into easy chairs for patients with arthritis. A survey instigated to define seating problems had revealed that the biggest problem that a patient with arthritis faces with his easy chair at home is getting out of it. ${ }^{1}$ Because the suggestions of previous workers, which are often subjective, do not seem to have solved the problems of rising from a chair, ${ }^{2}$ an objective measure of ease of rising was sought. The present study examined leg muscle activity electromyographically,

EMG cannot be used to determine quantitatively the muscle forces involved in any activity. However, it was thought that it might be used to determine any differences in phasic muscle activity, or gross differences in amount of activity, that could be related to a crude scale, when rising from different chairs. If such differences do occur and could be thus determined, and if they are related to ease of rising, then EMG could be used as an objective measure of ease of rising.

\section{Subjects and methods}

An initial study was carried out to determine which

Accepted for publication 7 February 1983.

Correspondence to Professor V. Wright, Rheumatology and Rehabilitation, School of Medicine, 36 Clarendon Road, Leeds LS2 9PJ. muscles were active during rising from a chair. Six muscles were investigated in 2 normal males, aged 21 and 28 years. In a further investigation 3 muscle groups of each leg were then studied in 7 subjects. These included 2 normal females and one normal male. The remaining subjects were 4 patients with arthritis, for whom the diagnoses were: subject 1 , female, Still's disease; subject 2 , female, rheumatoid arthritis, particularly affecting the left knee; subject 3 , female, rheumatoid arthritis, including flexion deformities at both knees; and subject 4 , male, bilateral hip replacement and bilateral knee replacement, rheumatoid hands.

\section{PROCEDURE}

The objective of these experiments was to monitor the activity of leg muscles and the angles of the lower limbs while the subjects rose from a chair, stood, then sat down again.

A detailed study was carried out initially on normal subjects, in whom 6 muscles of the right leg were monitored-gastrocnemius, tibialis anterior, rectus femoris, sole us, biceps femoris, and glute us maximus. This established which of these muscle groups were active. It was considered important to monitor both legs of the arthritic patients, since, because of their conditions, they might not use both legs in the same way or to the same degree when rising. As the EMG equipment had only 6 channels, this limited the number of muscles that could be monitored simul- 
taneously. Therefore in the main study 3 muscle groups on both legs were monitored, namely, the quadriceps, hamstrings, and gluteal muscle groups. These had been shown in the initial study to be the major muscles involved in rising.

The variables in chair design studied were: (1) changing the seat to floor height $(420 \mathrm{~mm}$ and 595 $\mathrm{mm}) ;(2)$ rising without the aid of armrests; (3) rising with the aid of armrests; (4) rising with the feet in a normal, comfortable position; (5) rising with the feet set back under the chair.

To carry out these studies the experimental system shown in Fig. 1 was used. To monitor the activity of the leg muscles a pair of nickel-silver surface electrodes were placed over each muscle under study. A rigorous cleaning procedure was followed to ensure the minimum skin resistance. The EMG signals from the muscles were led into an ultraviolet (UV) recorder from the main amplifier units.

To monitor the lower limb angles a polarised light goniometer (polgon) was used with the initial subjects. However, most of the subjects were monitored by means of a Locam high-speed cine camera at 30 frames/s. This had the advantage over the polgon of avoiding having to position and attach the light sensors and power pack belt. To the arthritic patients, particularly those who were also elderly, doing this was an added complication and an activity which sometimes detached the EMG electrodes.

To relate the muscle activity to each phase of rising, the camera and the EMG equipment were linked by a specially constructed electronic device.

There were particular problems in carrying out an EMG study with arthritic and elderly patients, espe-

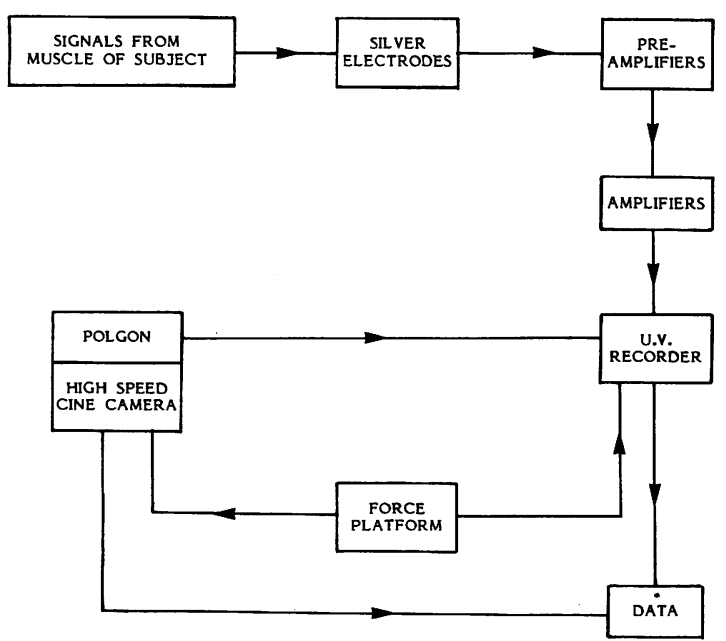

Fig. 1 Experimental system. cially in attaching the electrodes. It took several minutes to clean the skin with abrasive paper and alcohol, to attach adhesive stickers to the electrodes and place them on the skin, and to inject electrode gel into the electrodes. These subjects found it tiring and painful to stand for even this short period of time. To some extent this was solved by their lying on a bed while the hamstring and gluteal monitoring electrodes were attached. Care had to be taken, however, to prevent detachment of the electrodes as the patients moved off the bed and were assisted over to the EMG equipment. Although the normal subjects rose from the chair in a smooth, clean movement, the arthritics tended to drag their buttocks along the seat before starting to rise, so extra care had to be taken to prevent the hamstring and gluteal monitoring electrodes from being pulled off. Placing a length of nonconducting adhesive tape over each pair of electrodes helped to alleviate this problem. A towel was also placed on the seat to prevent the subject sticking to the chair owing to perspiration and detaching the electrodes when rising from it. If the electrodes had to be reattached, this created problems as the skin became sticky from perspiration and electrode gel. Another problem was that it was noticeably more difficult to attach electrodes to elderly subjects and to obtain a clear electrical signal, probably owing to a difference between the older and younger subjects in the quality of skin.

\section{Analysis of results.}

For ease of interpretation EMG signals were traced from the UV traces in block form to represent no muscle activity, a little muscle activity, and great muscle activity. Limb angles were taken from the cine film or polgon trace and synchronised with the UV recordings.

\section{Results}

General pattern of muscle activity. An example of a typical set of results is shown in Fig. 2. The pattern of leg muscle activity when rising from a chair was as follows. While the subjects were sitting, there was no activity in the muscles monitored. As the rising activity was initiated and subjects lost contact with the seat, the rectus femoris became active as an extensor of the knee and a flexor of the hip. The gluteus maximus, biceps femoris, and tibialis anterior became active simultaneously with the rectus femoris. As the subjects approached, and finally attained, a standing position; all these muscles except the biceps femoris became inactive. As the upright phase was approached, the soleus and gastrocnemius became active and remained so during standing. The pattern for sitting down was the reverse of that for 


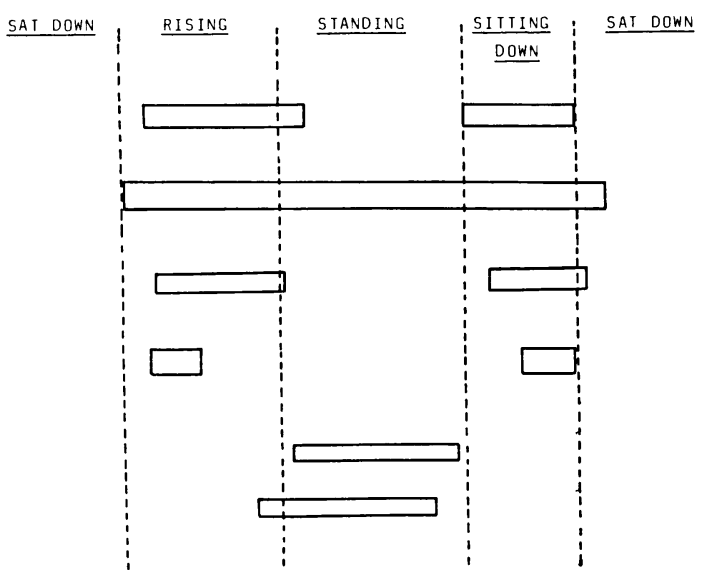

standing up. These results were in keeping with previous findings for normal subjects. ${ }^{34}$

Patients with arthritis. Patients with arthritis did not show such a clear 'on-off' pattern as normal subjects, but in general the pattern of muscle activity corresponding to the limb angles when rising from a chair was the same for the 2 groups.

Changing chair design. For both normal and arthritic subjects the pattern of muscle activity did not vary with the use of armrests or with changes in seat height or foot position. For some subjects small differences in amplitude of the EMG signal between the right and left legs were recorded. For example, arthritic subject 2 appeared to use her right leg more than her left (which was more severely affected by rheumatoid arthritis). However, small differences in amplitude could be due to variations in skin resistance or placement of the electrodes. It must be remembered that all these arthritic subjects were able to rise from the chair in a fairly conventional way, not using the contrived methods that patients more severely disabled by arthritis often use.

\section{Discussion}

The practical difficulties involved in carrying out EMG studies with arthritic or elderly subjects make results difficult to obtain. Nevertheless, EMG is a useful tool for determining the pattern of leg muscle activity during rising for both arthritic and normal subjects. No differences were found in the pattern of muscle activity when height of seat, position of feet, or use of arms was altered. EMG with surface electrodes is not sensitive enough to detect variations in ease of rising. The use of needle electrodes cannot be

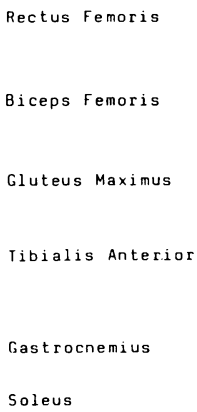

Tibialis Anterior

Gastrocnemius Soleus rising from a chair. considered owing to the pain and probable muscle damage due to gross muscle movements.

However, the variation in hip and knee angles, observation of subjects, and the comments from subjects all support the view that the dimensions and other characteristics of a chair, and the method employed to rise from it, greatly influence the ease of rising.

EMG cannot be used as a means of objectively assessing changes in rising caused by chair design. It does have a useful role to play in the development of more appropriate methodology and the assessment of its application to different groups of patients. An example of such an objective measure is that of joint force analysis and the analysis of muscle tensions, as has been developed in complementary studies. ${ }^{5}$

The authors are grateful to the Department of Health and Social Security, the Arthritis and Rheumatism Council, and the Emmandjay Trust for financial support. Thanks are also extended to M. Pullan and B. Whitham for technical assistance. and to A. Moreton for transporting patients. We also express our appreciation to friends and patients who volunteered as subjects and without whom this study could not have been conducted.

\section{References}

1 Munton J S, Ellis M I, Chamberlain M A, Wright V. An investigation into the problems of easy chairs used by the arthritic and the elderly. Rheumatol Rehabil 1981; 20: 164-73.

2 Munton J S. An overview of research on seating. Engineering Med 1982; 11: 107-10.

3 Kelley D L, Dainis A, Wood G K. Mechanics and muscular dynamics of rising from a seated position. In: Komi P V, ed. International series on biomechanics. Baltimore: University Park Press, 1976: 1B, Biomechanics V-B.

4 Ellis M I, Seedhom B B, Amis A A, Wright V, Dowson D. Forces in the knee joint whilst rising from normal and motorised chairs. Engineering Med 1979; 8: 33-40.

5 Ellis $\mathrm{M}$ I. Forces in the human knee during rising from a chair. PhD thesis. University of Leeds, 1982. 